\title{
Is the objective food environment associated with perceptions of the food environment?
}

\author{
Lauren K Williams*, Lukar Thornton, Kylie Ball and David Crawford \\ Centre for Physical Activity and Nutrition Research, School of Exercise and Nutrition Sciences, Deakin University, \\ 221 Burwood Highway, Burwood, Victoria 3125, Australia
}

Submitted 15 March 2011: Accepted 14 June 2011: First published online 11 August 2011

\begin{abstract}
Objective: The present study examined whether objective measures of the food environment are associated with perceptions of the food environment and whether this relationship varies by socio-economic disadvantage.

Design: The study is a cross-sectional analysis of self-report surveys and objective environment data. Women reported their perceptions on the nutrition environment. Participants' homes and food stores were geocoded to measure the objective community nutrition environment. Data on the average price and variety of fruit and vegetables were used to measure the objective consumer nutrition environment. Setting: The study was conducted in Melbourne, Australia, in 2003-2004.

Subjects: Data presented are from a sample of 1393 women aged 18-65 years. Results: Overall the match between the perceived and objective environment was poor, underscoring the limitations in using perceptions of the environment as a proxy for the objective environment. Socio-economic disadvantage had limited impact on the relationship between perceived and objective nutrition environment.

Conclusions: Further research is needed to understand the determinants of perceptions of the nutrition environment to enhance our understanding of the role of perceptions in nutrition choices and drivers of socio-economic inequalities in nutrition.
\end{abstract}

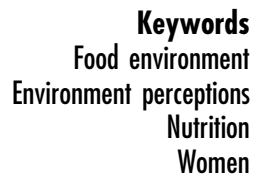

There is clear evidence of socio-economic gradients in dietary intakes among women; disadvantaged women, including those with low incomes, low education and those residing in socio-economically disadvantaged neighbourhood areas, generally have higher intakes of energy and fat and lower intakes of fruit and vegetables than those who are less disadvantaged ${ }^{(1-5)}$. Negative perceptions of the food environment have been found to be associated with these socio-economic inequalities in diet. For instance, compared with more advantaged women, socio-economically disadvantaged women have been demonstrated to have more negative perceptions of price, access and availability of healthy foods in their local neighbourhoods, and these perceptions are associated with purchasing and consumption of less nutritious foods ${ }^{(6-9)}$. Although the role of cognitions ${ }^{(10)}$ and environmental opportunities ${ }^{(11,12)}$ in predicting and changing health behaviours is well established, theoretical models and studies of environments and eating behaviours have only recently considered specific food environment perceptions as an important determinant in mediating the pathway between the objective nutrition environment and eating patterns ${ }^{(13)}$. Little is known about what determines environmental perceptions or why women of low socioeconomic position (SEP) perceive their food environment as more negative than those of higher SEP.

Plausibly, negative perceptions of the food environment held by disadvantaged women are attributable to the neighbourhoods in which they reside actually having a less healthy food environment. For instance, increasing evidence demonstrates that those residing in disadvantaged areas have less access to supermarkets, greater access to fast food and convenience stores, and fewer healthy food choices available in stores ${ }^{(14-18)}$. However, evidence of socio-economic differences in the food environment remains equivocal, particularly among studies conducted outside the USA, where little difference in access and availability of food stores is observed between neighbourhoods of varying levels of disadvantage ${ }^{(16,19-22)}$ and limited evidence exists that poorer diet maintained by low SEP women is attributable to less supportive food environments ${ }^{(23)}$.

Although much of the research focuses on area-level SEP differences in the food environment, individual-level SEP can also influence differences in perceptions of the nutrition environment. For instance, women with low education may 
place less value on nutrition and health, resulting in lower motivation to allocate a higher proportion of their food budget on healthy foods, and hence perceive the same priced food as less affordable than higher educated women. Further, low-SEP women may have further barriers placed on their ability to interact with their food environment (e.g. may not have access to a vehicle to support purchases of higher volume and variety, resulting in more negative perceptions of healthy food accessibility).

Given that environmental perceptions appear to play an important role to shaping women's diets, it is important to understand factors that influence these perceptions in order to determine the best avenue for intervention. Furthermore, if perceptions of the nutrition environment are not determined by the objective nutrition environment, implementing changes to the food environment to improve nutrition (e.g. by reducing the price of healthy food for disadvantaged groups) may be ineffective. More research is needed to examine the associations between objective and perceived food environments and whether the relationship between the objective food environment and the perceived food environment varies by socioeconomic disadvantage.

The aims of the present study were to: (i) examine whether objective measures of the food environment are associated with environmental perceptions; and (ii) to assess whether individual- and area-level socio-economic disadvantage moderate the association between the objective and perceived food environment.

\section{Methods}

\section{Sample}

Analyses presented are from a sample of 1393 women who were part of a study conducted in 2003-2004 that assessed socio-economic and neighbourhood inequalities in women's physical activity, diet and obesity (SESAW study) ${ }^{(5)}$. A total of 2400 questionnaires were posted to women aged 18-65 years, sampled randomly from fortyfive neighbourhoods of low, mid and high levels of socioeconomic disadvantage. Of these, 1136 (47.3\%) women completed the survey. A second independent sample ( $n$ 2400) was invited to complete a separate physical activity survey and participants completing that survey were asked if they were also willing to complete the dietary survey. This yielded an additional 444 participants. Of the 1580 women who provided complete questionnaires, thirteen participants were excluded as they had moved address prior to completing the survey and were no longer eligible as they resided outside the study suburbs. A further 174 women were excluded due to missing data (as only participants with complete data on all variables were included in analyses). Response rates for low, mid and high levels of socio-economic disadvantage were $33 \%, 38 \%$, and 29\%, respectively.

\section{Procedures}

The study was approved by the Deakin University Human Research Ethics Committee. According to the methods described by Dillman ${ }^{(24)}$, women were initially mailed a letter advising them they had been selected to take part in a study of women's health behaviours and they would shortly receive a survey. One week later a self-completion dietary questionnaire was posted out to 2400 women and a physical activity questionnaire was sent to a separate sample of 2400 women. A small incentive was included in the initial survey package (\$AU 1 lottery ticket). Participants who did not respond after three weeks were sent a reminder postcard, and a second reminder was sent after a further three weeks with a replacement survey package.

\section{Measures}

Outcome measures: perceived consumer and

community nutrition environment

Based on Glanz et al.'s ${ }^{(13)}$ model of nutrition environments, environmental variables were categorised as elements of either the community nutrition environment (type, location and accessibility of food stores) or consumer nutrition environment (in-store variables such as availability and price). Three community and three consumer nutrition environment items were used to measure the perceived food environment. For the community environment items, participants were asked to answer 'yes', 'no', or 'don't know' to the questions 'Are the following within walking distance of your home?: Supermarket; Fruit/vegetable store; Fast food restaurant'. Response items 'no' and 'don't know' were collapsed into one response category. For the consumer environment items, participants were asked to rate how much they agreed or disagreed with the following three statements: 'At the shops where I buy fruits and vegetables, the variety of fresh fruits and vegetables is limited', 'I do not buy many fruits because they cost too much' and 'I do not buy vegetables because they cost too much'. The five response categories were: 'strongly disagree' (scored 1), 'disagree' (2), 'neither agree nor disagree' (3), 'agree' (4) and 'strongly agree' (scored 5). Responses were dichotomised into do not agree (score $\leq 3$ ) and agree (score $\geq 4$ ).

Predictor measures: objective community nutrition and consumer environment, individual and neighbourbood socio-economic position

Objective nutrition environment. For the objective community environment variables, data on locations of greengrocers, major supermarkets and fast-food restaurants in and immediately surrounding the forty-five neighbourhoods were sourced through extensive searches of online telephone directories, local council/government websites and company websites in 2004. Greengrocers were defined as stores that primarily sold fresh fruit and vegetables. Supermarkets were identified as belonging to one of the five large supermarket chains within Australia. 
Nine major fast-food restaurant chains were included: Dominos, Kentucky Fried Chicken (KFC), Hungry Jacks, McDonalds, Nandos, Pizza Haven, Pizza Hut, Red Rooster and Subway. Geocoding of participants and food stores was undertaken using a Geographic Information System (ArcView 3·3; ESRI, Redlands, CA, USA) and overlaid with the road network (VicMap Transport version 2004; owned and supplied by the State of Victoria, Australia). Stores of each type that were within $800 \mathrm{~m}$ of road network distance from each participant's household were considered within walking distance (equivalent to approximately $10 \mathrm{~min}$ of walking one way based on standard $5 \mathrm{~km} / \mathrm{h}$ walking pace). This definition of 'walkable distance' is consistent with previous research that has used an objective cut-off to classify walking distance to neighbourhood food stores ${ }^{(25)}$. Participants were classified as having or not having each store within this $800 \mathrm{~m}$ proximity.

Objective consumer environment. The objective consumer environment variables were based on the cost and variety of fruit and vegetables in local stores. Data were gathered by trained research assistants during store audits on the availability and price of fifteen commonly consumed fruits and twenty-three vegetables in 134 stores identified as being within the boundaries of the forty-five neighbourhoods. From this list of thirty-eight fruit and vegetable items, the average number of fruit and vegetable items available in each store was calculated and this was used to measure fruit and vegetable variety. A median split of the average number of fruit and vegetables was used to classify high (scored 1) and low (scored 0) fruit and vegetable variety.

Data were also collected on the price per kilogram, or the price per item for individually priced items (e.g. mangoes), of the cheapest item available within each store. Prices per item were converted to price per kilogram by dividing an item's price by its typical weight, obtained from a comprehensive food list software package (FoodWorks Professional version 2007; Xyris Software, Brisbane, Australia). The mean price for each fruit and vegetable item across all stores was calculated. For each item available within a store, the cost difference between that item and the overall mean price for that item across all stores was calculated. This 'mean difference' was summed for all items in a store and divided by the number of items available, resulting in a single price figure for each store. This single price figure was used to classify the 'price' of fruit and vegetables whereby fruit and vegetables priced at or below the average for all stores were classified as 'inexpensive' (scored 0), and fruit and vegetables priced above average were classified as 'expensive' (scored 1) ${ }^{(23)}$.

Individual SEP: individual educational level. Education was used as the individual-level indicator of SEP in the present study. Education was defined as self-reported highest level of education attained in three categories: (i) no formal qualifications/up to year 10 (low individuallevel SEP); (ii) year 12/trade/apprenticeship/certificate/ diploma (medium individual-level SEP); or (iii) university degree/higher degree (high individual-level SEP). This definition of SEP was considered the most appropriate personal indicator of socio-economic disadvantage for women, for whom occupation, income and employment status often fluctuate during childbearing years, while educational attainment remains relatively stable.

Neighbourbood SEP. Classification of neighbourhood level socio-economic position was conducted using Socio-Economic Index for Areas (SEIFA) scores that are based on 2001 census data from the Australian Bureau of Statistics. SEIFA scores classify suburbs based on relative disadvantage (which considers the proportion of residents with low income, low educational attainment, and unskilled occupations) ${ }^{(26)}$. All suburbs within the study area (a $30 \mathrm{~km}$ radius of the Central Business District) were ranked according to SEIFA score and grouped into tertiles of socio-economic disadvantage. A random sample of fifteen suburbs from each SEIFA tertile was selected. Based on suburb of residence, participants were scored as low, medium or high area-level SEP.

\section{Statistical analyses}

Statistical analyses were conducted using the statistical software packages SPSS version $17 \cdot 0$ (SPSS Inc., Chicago, IL, USA) and STATA version $11 \cdot 0$ (StataCorp., College Station, TX, USA). Differences in environmental perceptions according to individual- and neighbourhood-level SEP were assessed using the $\chi^{2}$ statistic. Level of agreement between the objective and perceived environment was assessed using the $\kappa$ statistic where values of $0 \cdot 5,0 \cdot 7$ and 0.8 were regarded as moderate, good and very good agreement, respectively ${ }^{(27)}$. Logistic regression analysis (adjusting for age and neighbourhood-level clustering of the sample) was used to assess the main effects and interactions between objective food environment measures, SEP and environment perceptions. Adjustment for education in logistic regression models that assessed area-level SEP was also employed to account for the temporal relationship between education and area-level SEP (i.e. a large amount of area-level SEP is likely explained by individual education). Cost and variety data were not available for all respondents ( $n$ 195) as withinstore data were collected only for stores that fell within the forty-five neighbourhood boundaries. As a result, we do not have within-store information for participants who did not have a store in their suburb of residence or if the closest store was more than $3 \mathrm{~km}$ from their home, as a distance beyond this was deemed not accessible.

\section{Results}

The mean age of the sample was 42 (SD 12.6) years. Just over a third of the sample were tertiary educated $(35 \cdot 1 \%)$ and $22 \cdot 7 \%$ had no formal education. The sample was roughly evenly divided into those residing in 
neighbourhoods of low (30.4\%), medium (36.9\%) and high $(31 \cdot 8 \%)$ SEP.

Table 1 shows that a lower proportion of those residing in low-SEP neighbourhoods perceived greengrocers and fast-food stores to be within walking distance and a higher proportion of women residing in low-SEP neighbourhoods perceived less variety and higher prices of fruit and vegetables in their local food environment. A significantly higher proportion of women with low individual-level SEP perceived the cost of fruit and vegetables to be high, compared with women of medium and high individual-level SEP.

Table 2 shows the correspondence between perceived and objectively assessed features of the food environment. There was substantial, albeit low, agreement between objective and perceived indices for supermarket and greengrocer store access, with approximately 50\% of women having perceptions which matched objective mea- sures. Correspondence between perceived and objective measures of the remaining aspects of the food environment was poor.

Table 3 shows results for associations between objective measures of the food environment and perceptions of the food environment (Model 1), and the effect of individuallevel and area-level SEP on these relationships (Model 2). Results indicated that women who, according to objective measures, had access to supermarkets were three-and-ahalf times more likely than those who did not have access to perceive that they had a supermarket within walking distance. Women who had access to a greengrocer were five times more likely, than those who did not have access, to perceive they had a greengrocer within walking distance. None of the other objective measures of the food environment (access to fast-food stores, variety and cost of fruit and vegetables) were associated with perceptions of the food environment (Table 3, Model 1).

Table 1 Differences by individual and neighbourhood disadvantage in perceptions of the local food environment among women aged 18-65 years, Melbourne, Australia, 2003-2004

\begin{tabular}{|c|c|c|c|c|c|c|c|c|}
\hline \multirow[b]{3}{*}{ Environment perceptions } & \multicolumn{3}{|c|}{ Individual-level SEP ( $n$ 1393) } & & \multicolumn{3}{|c|}{ Area-level SEP ( $n$ 1393) } & \multirow[b]{3}{*}{$P$} \\
\hline & $\begin{array}{c}\text { Low } \\
(n \text { 320) }\end{array}$ & $\begin{array}{c}\text { Medium } \\
(n \text { 560) }\end{array}$ & $\begin{array}{l}\text { High } \\
(n \text { 513) }\end{array}$ & \multirow[b]{2}{*}{$P$} & $\begin{array}{c}\text { Low } \\
(n \text { 454) }\end{array}$ & $\begin{array}{c}\text { Medium } \\
(n \text { 535) }\end{array}$ & $\begin{array}{l}\text { High } \\
(n \text { 404) }\end{array}$ & \\
\hline & $\%$ & $\%$ & $\%$ & & $\%$ & $\%$ & $\%$ & \\
\hline \multicolumn{9}{|l|}{ Community environment } \\
\hline Perceive supermarket(s) within walking distance & $66 \cdot 9$ & $68 \cdot 2$ & $64 \cdot 9$ & 0.52 & $63 \cdot 6$ & $68 \cdot 0$ & $67 \cdot 8$ & $0 \cdot 30$ \\
\hline Perceive fruit and vegetable store(s) within walking distance & $63 \cdot 1$ & $65 \cdot 5$ & $67 \cdot 8$ & $0 \cdot 37$ & $57 \cdot 7$ & $61 \cdot 5$ & $78 \cdot 2$ & 0.00 \\
\hline Perceived fast food outlet(s) within walking distance & $64 \cdot 1$ & $67 \cdot 1$ & $70 \cdot 5$ & $0 \cdot 14$ & $65 \cdot 8$ & $62 \cdot 1$ & $75 \cdot 9$ & 0.00 \\
\hline \multicolumn{9}{|l|}{ Consumer environment } \\
\hline Perceived limited variety of fruit and vegetables & $15 \cdot 3$ & $12 \cdot 3$ & $10 \cdot 1$ & 0.09 & $14 \cdot 4$ & $13 \cdot 3$ & $9 \cdot 0$ & 0.04 \\
\hline Perceived cost of fruit too high & $15 \cdot 6$ & $13 \cdot 7$ & $8 \cdot 4$ & 0.00 & $16 \cdot 8$ & $11 \cdot 4$ & $8 \cdot 8$ & 0.00 \\
\hline Perceived cost of vegetables too high & $11 \cdot 6$ & $8 \cdot 6$ & $3 \cdot 3$ & 0.00 & $10 \cdot 6$ & $7 \cdot 1$ & $4 \cdot 6$ & 0.00 \\
\hline
\end{tabular}

SEP, socio-economic position.

Table 2 Correspondence between perceived and objectively assessed aspects of the local food environment (percentage of women with presence/absence of objective measure) among women ( $n$ 1393) aged 18-65 years, Melbourne, Australia, 2003-2004

\begin{tabular}{|c|c|c|c|}
\hline \multirow[b]{2}{*}{ Objective food environment } & \multicolumn{2}{|c|}{ Perceptions } & \multirow[b]{2}{*}{$\kappa$} \\
\hline & Yes & No & \\
\hline Yes & $20 \cdot 3$ & $3 \cdot 6$ & \\
\hline No & $46 \cdot 4$ & $29 \cdot 7$ & \\
\hline Fruit and vegetable store within $800 \mathrm{~m}$ from home & & & $0 \cdot 19^{*}$ \\
\hline No & $43 \cdot 9$ & $31 \cdot 0$ & \\
\hline Fast-food store within $800 \mathrm{~m}$ from home & & & $-0 \cdot 00$ \\
\hline Yes & $14 \cdot 1$ & $6 \cdot 8$ & \\
\hline No & $53 \cdot 6$ & $25 \cdot 5$ & \\
\hline Cost of fruit high & & & -0.03 \\
\hline Yes & $6 \cdot 4$ & $54 \cdot 1$ & \\
\hline Limited variety of fruit and vegetables & & & -0.01 \\
\hline Yes & $4 \cdot 9$ & $38 \cdot 9$ & \\
\hline No & $6 \cdot 9$ & $49 \cdot 3$ & \\
\hline
\end{tabular}

${ }^{\star} P<0.001$. 
Table 3 Adjusted odds ratios and $95 \%$ confidence intervals for associations between the objective and perceived food environment (Model 1 ) and interactions by area- and individual-level SEP (Model 2) among women ( $n$ 1393) aged 18-65 years, Melbourne, Australia, 2003-2004

\begin{tabular}{|c|c|c|c|c|c|c|c|}
\hline \multirow[b]{2}{*}{ Socio-economic and objective food environment correlates } & \multirow{2}{*}{$\begin{array}{l}\text { Perception of the food } \\
\text { environment }\end{array}$} & \multicolumn{2}{|c|}{ Model 1} & \multirow[b]{2}{*}{$P$} & \multicolumn{2}{|c|}{ Model 2} & \multirow[b]{2}{*}{$P$} \\
\hline & & OR & $95 \% \mathrm{Cl}$ & & OR & $95 \% \mathrm{Cl}$ & \\
\hline \multicolumn{8}{|l|}{ Community environment ( $n$ 1393) } \\
\hline $\begin{array}{l}\text { Supermarket access within } 800 \mathrm{~m} \text { from home } \\
\text { Supermarket access } \times \text { individual-level SEP }\end{array}$ & $\begin{array}{l}\text { Perceive supermarket within } \\
\text { walking distance }\end{array}$ & $3.56+$ & $2 \cdot 40,5 \cdot 28$ & 0.00 & & & \\
\hline $\begin{array}{l}\text { Supermarket access and medium individual-level SEP } \\
\text { Supermarket access and high individual-level SEP }\end{array}$ & & & & & $\begin{array}{l}0.58 t \\
0.32 t\end{array}$ & $\begin{array}{l}0.22,1 \cdot 55 \\
0 \cdot 14,0.97\end{array}$ & $\begin{array}{l}0.28 \\
0.02\end{array}$ \\
\hline \multicolumn{8}{|l|}{ Supermarket access $\times$ area-level SEP } \\
\hline Supermarket access and medium area-level SEP & & & & & $1 \cdot 92 \ddagger$ & $0 \cdot 75 \cdot 4.94$ & $0 \cdot 18$ \\
\hline Supermarket access and low area-level SEP & & & & & $1 \cdot 20 \ddagger$ & $0.51,2 \cdot 85$ & 0.68 \\
\hline $\begin{array}{l}\text { Greengrocer access within } 800 \mathrm{~m} \text { from home } \\
\text { Greengrocer access } \times \text { individual-level SEP }\end{array}$ & $\begin{array}{l}\text { Perceive greengrocer within } \\
\text { walking distance }\end{array}$ & $5 \cdot 10+$ & $3 \cdot 27,7 \cdot 84$ & $0 \cdot 00$ & & & \\
\hline $\begin{array}{l}\text { Greengrocer access and medium individual-level SEP } \\
\text { Greengrocer access and high individual-level SEP }\end{array}$ & & & & & $\begin{array}{l}0.98 t \\
0.56 t\end{array}$ & $\begin{array}{l}0 \cdot 44,2 \cdot 20 \\
0 \cdot 22,1 \cdot 42\end{array}$ & $\begin{array}{l}0.96 \\
0.22\end{array}$ \\
\hline \multicolumn{8}{|l|}{ Greengrocer access $\times$ area-level SEP } \\
\hline $\begin{array}{l}\text { Greengrocer access and medium area-level SEP } \\
\text { Greengrocer access and low area-level SEP }\end{array}$ & & & & & $\begin{array}{l}1 \cdot 81 \ddagger \\
0.81 \ddagger\end{array}$ & $\begin{array}{l}0.63,5 \cdot 22 \\
0.33,1.98\end{array}$ & $\begin{array}{l}0.27 \\
0.64\end{array}$ \\
\hline $\begin{array}{l}\text { Fast-food access within } 800 \mathrm{~m} \text { from home } \\
\text { Fast-food access } \times \text { individual-level SEP }\end{array}$ & $\begin{array}{l}\text { Perceive fast-food restaurant } \\
\text { within walking distance }\end{array}$ & $0 \cdot 98+$ & $0 \cdot 65,1 \cdot 47$ & $0 \cdot 91$ & & & \\
\hline Fast-food access and medium individual-level SEP & & & & & $0 \cdot 79+$ & $0.39,1.58$ & 0.51 \\
\hline Fast-food access and high individual-level SEP & & & & & $1.06+$ & $0 \cdot 46,2 \cdot 41$ & 0.90 \\
\hline \multicolumn{8}{|l|}{ Fast-food access $\times$ area-level SEP } \\
\hline Fast-food access and medium area-level SEP & & & & & $0 \cdot 81 \ddagger$ & $0 \cdot 35,1 \cdot 86$ & 0.63 \\
\hline Fast-food access and low area-level SEP & & & & & $0.92 \ddagger$ & $0.42,2 \cdot 04$ & $0 \cdot 84$ \\
\hline \multicolumn{8}{|l|}{ Consumer environment ( $n$ 1198) } \\
\hline $\begin{array}{l}\text { High variety of fruit and vegetables at local stores } \\
\text { High variety of fruit and vegetables } \times \text { individual-level SEP }\end{array}$ & $\begin{array}{l}\text { Perceived high variety of fruit } \\
\text { and vegetables }\end{array}$ & $0 \cdot 89+$ & $0 \cdot 63,1 \cdot 26$ & 0.51 & & & \\
\hline High variety of fruit and vegetables and medium individual-level SEP & & & & & $0.71+$ & $0 \cdot 25,2 \cdot 01$ & 0.52 \\
\hline High variety of fruit and vegetables and high individual-level SEP & & & & & $0.73+$ & $0 \cdot 26,2 \cdot 07$ & 0.55 \\
\hline \multicolumn{8}{|l|}{ High variety of fruit and vegetables $\times$ area-level SEP } \\
\hline High variety of fruit and vegetables and medium area-level SEP & & & & & $0.93 \ddagger$ & $0 \cdot 40,2 \cdot 17$ & 0.86 \\
\hline High variety of fruit and vegetables and low area-level SEP & & & & & $0.69 \ddagger$ & $0.23,2 \cdot 04$ & 0.50 \\
\hline $\begin{array}{l}\text { Cost of fruit high } \\
\text { Cost of fruit high } \times \text { individual-level SEP }\end{array}$ & Perceived cost of fruit & $0 \cdot 71+$ & $0 \cdot 48,1 \cdot 06$ & 0.09 & & & \\
\hline $\begin{array}{l}\text { Cost of fruit high } \times \text { individual-level SEP } \\
\text { Cost of fruit high and medium individual-level SEP }\end{array}$ & & & & & $0.97 \dagger$ & $0 \cdot 44,2 \cdot 15$ & 0.94 \\
\hline Cost of fruit high and high individual-level SEP & & & & & $0.63+$ & $0.28,1 \cdot 44$ & 0.28 \\
\hline \multicolumn{8}{|l|}{ Cost of fruit high $\times$ area-level SEP } \\
\hline Cost of fruit high and medium area-level SEP & & & & & $0.93 \ddagger$ & $0 \cdot 16,5 \cdot 41$ & 0.94 \\
\hline Cost of fruit high and low area-level SEP & & & & & $0.78 \ddagger$ & $0 \cdot 14,4 \cdot 31$ & $0 \cdot 77$ \\
\hline $\begin{array}{l}\text { Cost of vegetables high } \\
\text { Cost of vegetables high } \times \text { individual-level SEP }\end{array}$ & $\begin{array}{l}\text { Perceived cost of vegetables } \\
\text { too high }\end{array}$ & $0 \cdot 87+$ & $0.51,1 \cdot 47$ & $0 \cdot 60$ & & & \\
\hline Cost of vegetables high and medium individual-level SEP & & & & & $0 \cdot 68+$ & $0 \cdot 25,1 \cdot 83$ & 0.45 \\
\hline Cost of vegetables high and high individual-level SEP & & & & & $0.94 \dagger$ & $0.22,3.95$ & 0.93 \\
\hline \multicolumn{8}{|l|}{ Cost of vegetables high $\times$ area-level SEP } \\
\hline Cost of vegetables high and medium area-level SEP & & & & & $1 \cdot 78 \ddagger$ & $0.58,5 \cdot 50$ & 0.32 \\
\hline Cost of vegetables high and low area-level SEP & & & & & $1 \cdot 47 \ddagger$ & $0.47,4.54$ & 0.51 \\
\hline
\end{tabular}

SEP, socio-economic position.

tModel adjusted for age and clustering of suburbs.

¥Model adjusted for age, clustering of suburbs and education. 
Table 4 Stratified odds ratios and confidence intervals for significant interactions among women ( $n$ 1393) aged 18-65 years, Melbourne, Australia, 2003-2004

\begin{tabular}{|c|c|c|c|c|c|}
\hline $\begin{array}{l}\text { Objective food environment } \\
\text { variable }\end{array}$ & $\begin{array}{l}\text { Perception of the food } \\
\text { environment }\end{array}$ & Disadvantage index & OR & $95 \% \mathrm{Cl}$ & $P$ \\
\hline \multicolumn{6}{|l|}{ Community environment } \\
\hline $\begin{array}{l}\text { Supermarket access within } \\
800 \mathrm{~m} \text { from home }\end{array}$ & $\begin{array}{l}\text { Perceive supermarket within } \\
\text { walking distance }\end{array}$ & $\begin{array}{l}\text { Low individual-level SEP† } \\
\text { Medium individual-level SEP† } \\
\text { High individual-level SEP† }\end{array}$ & $\begin{array}{l}7 \cdot 87 \\
4 \cdot 51 \\
2 \cdot 47\end{array}$ & $\begin{array}{l}3 \cdot 11,19 \cdot 90 \\
2 \cdot 61,7 \cdot 80 \\
1 \cdot 62,3 \cdot 76\end{array}$ & $\begin{array}{l}0.00 \\
0.00 \\
0.00\end{array}$ \\
\hline
\end{tabular}

SEP, socio-economic position.

tModel adjusted for age and clustering of suburbs.

Interaction analyses were conducted to assess whether individual-level and area-level SEP moderated the association between the objective and perceived food environment (Table 3, Model 2). Results indicated that individual-level SEP moderated the relationship between objective and perceived access to supermarkets. Stratified analyses revealed that women of low individual-level SEP who had access to a supermarket were almost eight times more likely to perceive they could walk to a supermarket, whereas women of high individual-level SEP who had access to a supermarket were only two-and-a-half times more likely to perceive they could walk to a supermarket (Table 4). For the remaining measures, the relationship between objective and perceived indices of the food environment did not differ by individual-level or area-level SEP.

\section{Discussion}

The aim of the present study was to examine whether objective measures of the nutrition environment were associated with perceptions of the nutrition environment and whether this relationship differed by socio-economic disadvantage. Results are consistent with previous stu$\operatorname{dies}^{(8,9)}$ in showing that perceptions of the nutrition environment differed by SEP; disadvantaged women were more likely to have negative perceptions of the nutrition environment than less disadvantaged women. However, while socio-economic inequalities in perceptions of the nutrition environment have been previously documented, to our knowledge, the present study is the first to assess the objective community and consumer environmental determinants of a range of nutrition environment perceptions, and also to test whether the association of perceived and objective nutrition environment variables differs by SEP. Overall, the level of agreement between the objective and perceived nutrition environment was relatively low, and with the exception of access to supermarkets and greengrocers, it appeared that the objective nutrition environment was not associated with perceptions of the nutrition environment. Furthermore, there was limited evidence that the relationship between the objective and perceived nutrition environment differed by SEP.
In terms of the community nutrition environment, consistent with previous research ${ }^{(28)}$, compared with women who did not have access to supermarkets and green grocers, women who had access to these stores were more likely to perceive they had a supermarket and greengrocer within walking distance. This finding was not observed for fast-food store access. We posit that differences in the store type and interactions women have with each type of food store may account for this finding. Supermarkets and greengrocers are regularly utilised to attain basic essentials, as well as core and more reasonably priced food items, whereas fast-food outlets represent sources of specific food choices, usually within a single cuisine type (e.g. hamburgers, chicken, etc.). It is therefore likely that women access supermarkets and greengrocers more regularly than specific fast-food outlets and are more aware of the presence and location of these more 'essential' food stores. The absence of a relationship between access to fast-food stores and perceived access to these stores may be attributed to a wider variation of fast-food stores accessed by women that was not captured in the current study, compared with supermarkets and greengrocers. For instance, some women may use fast-food stores that are close to work or school and therefore have limited awareness of the presence and location of fast-food stores close to home. In addition, fast-food purchasing may be indicative of less healthy behaviours generally, and therefore more likely to be accessed by women who are less physically active, resulting in nearby fast-food stores not being perceived by some women as 'walkable'.

It is interesting that the relationship between the objective and perceived community nutrition environment was moderated by individual-level SEP for access to supermarkets only. It is plausible that compared with more advantaged women, women with low individuallevel SEP rely more on stores such as supermarkets, where core foods are obtained and where food is often cheapest compared with convenience stores and smallchain supermarkets. When considering area-level SEP, although women in low-SEP areas maintained more negative perceptions of the community nutrition environment than those in medium- and high-SEP areas, area-level SEP differences in the relationship between the objective and perceived community nutrition environment were 
not observed. It is likely that other area-level environmental factors account for area-level SEP differences in perceptions of the community nutrition environment. For instance, individuals residing in low-SEP neighbourhoods may have access to fewer aesthetically pleasant or safe walking routes, or they may report less enjoyment of walking than individuals residing in high area-level SEP neighbourhoods. This could result in negative perceptions of aspects of the nutrition environment (e.g. perceiving stores as not being in walking distance). Overall, our results regarding the community nutrition environment suggest that although the match between perceived and objective measures is low, proximity to supermarkets and greengrocers is associated with increased likelihood of women accurately perceiving these stores as within walking distance.

The finding that perceptions of the consumer nutrition environment were largely unrelated to the objective nutrition environment could be a result of a poor match between our objective and perceived consumer environmental measures. For instance, prices of fruit and vegetables were dichotomised above or below the average for all items in all stores, and as such only measured one potential objective influence of perceived cost. This categorisation does not account for other 'costs' of fruit and vegetables potentially captured in women's perceptions of the nutrition environment and it is likely that a range of objective 'cost' measures is needed to best reflect perceptions of affordability. For instance, perceptions of price and availability of fruit and vegetables may also be influenced by overall budget and proportional spending (i.e. proportion of budget allocated to food), cost of fruit and vegetables relative to more inexpensive food, and other non-economic factors that impact on perceptions of cost and availability such as value for money, quality of produce and convenience. Plausibly, these explanations account for SEP differentials in perceptions that were not attributed to the objective consumer nutrition environment. For instance, decisions about the type of food to purchase on a restricted budget may mean that low-SEP women perceive fruit and vegetables to be expensive because more energy-dense foods are often available at a lower $\operatorname{cost}^{(29,30)}$, not because the cost of fruit and vegetables is higher at their store relative to other stores.

A discrepancy between perceived and objective assessments of the consumer environment may also be attributable to differences between stores measured in the current study and those utilised by participants (e.g. perceptions of variety of fruit and vegetables may be based on stores not included in the current study). Therefore, participants may be willing to shop in stores outside their local proximity to obtain better quality, lower-cost food.

The most notable limitation of the present study is the potential mismatch of objective and perceived environment measures. Despite the fact that our objective measures of food stores included the top five Australian supermarkets and nine major fast-food restaurants, items that assessed perceptions of food stores did not encompass these same restrictions. While we did not use field validations to verify our objective measures, we are confident that by acquiring data from multiple sources we have a comprehensive measure of the food stores located in our study areas. Previous research that relies solely on a single secondary data set sourced from commercial companies may be at greater risk of misrepresenting the food environment as inaccuracies are known to exist in these data sets $^{(31,32)}$. Furthermore, items that assessed perceptions did not quantify 'walking distance' as $800 \mathrm{~m}$ and plausibly wide variations in perceptions of what constitutes 'walking distance' exist.

Acknowledging these limitations, the present study shows that although disadvantaged women maintain poorer perceptions of the nutrition environment, the objective environment was generally unrelated to these perceptions. Although previous research has indicated a relationship between the observed neighbourhood food environment and satisfaction of availability of healthy produce $^{(33)}$, these results underscore the limitations in using matched perceptions of the environment in research studies as a proxy for the objective environment. Finally, there was limited evidence that differences in individual-level and area-level SEP influenced the relationship between the objective and perceived nutrition environment. In summary, while proximity to supermarkets and greengrocers may have a positive effect on perceptions and consumption of healthy food, our results suggest that nutrition promotion interventions targeting the consumer environment (e.g. healthy food incentives) should incorporate strategies to overcome negative perceptions about the cost and availability of fruit and vegetables and consider non-economic factors associated with the cost and variety of fruit and vegetables. Our results show that changing the price and availability of fruit and vegetables alone may not change perceptions or consumption of healthy food. Further research is needed to understand the determinants of perceptions of the nutrition environment to enhance our understanding of the drivers of socio-economic inequalities in nutrition.

\section{Acknowledgements}

The study on which this paper was based was funded by a National Heart Foundation of Australia grant-in-aid (GIA G 02M 0658) and an Australian Research Council Discovery Grant (DP0665242). L.K.W. and L.T. were supported by a National Health and Medical Research Council Capacity Building Grant (ID 425845). K.B. was supported by a National Health and Medical Research Council Senior Research Fellowship (ID 479513). D.C. was supported by a VicHealth Research Fellowship. The authors declare that there are no conflicts of interest. L.K.W. wrote the 
manuscript. L.T., D.C. and K.B. assisted with writing the manuscript. L.K.W. and L.T. conducted the statistical analyses. D.C. and K.B designed the SESAW study. The authors are grateful to Lena Stephens for assistance with data collection and management and to Rebecca Roberts for assistance with geospatial analyses.

\section{References}

1. De Irala-Estevez J, Groth M, Johannson L et al. (2000) A systematic review of socio-economic differences in food habits in Europe: consumption of fruit and vegetables. Eur J Clin Nutr 54, 706-714.

2. Giskes K, Turrell G, Patterson C et al. (2002) Socioeconomic differences among Australian adults in consumption of fruit and vegetables and intakes of vitamins A, C and folate. J Hum Nutr Diet 15, 375-385.

3. Lallukka T, Laaksonen M, Rahkonen O et al. (2007) Multiple socio-economic circumstances and healthy food habits. Eur J Clin Nutr 61, 701-710.

4. Pearson T, Russell J, Campbell MJ et al. (2005) Do 'food deserts' influence fruit and vegetable consumption? - A cross-sectional study. Appetite 45, 195-197.

5. Ball K, Crawford D \& Mishra G (2006) Socio-economic inequalities in women's fruit and vegetable intakes: a multilevel study of individual, social and environmental mediators. Public Health Nutr 9, 623-630.

6. Kamphuis CB, van Lenthe FJ, Giskes K et al. (2007) Perceived environmental determinants of physical activity and fruit and vegetable consumption among high and low socioeconomic groups in the Netherlands. Health Place 13, 493-503.

7. Inglis V, Ball K \& Crawford D (2008) Socioeconomic variations in women's diets: what is the role of perceptions of the local food environment? J Epidemiol Community Health 62, 191-197.

8. Kamphuis CB, Giskes K, de Bruijn GJ et al. (2006) Environmental determinants of fruit and vegetable consumption among adults: a systematic review. Br J Nutr $\mathbf{9 6}$, 620-635.

9. Giskes K, Van Lenthe FJ, Brug J et al. (2007) Socioeconomic inequalities in food purchasing: the contribution of respondent-perceived and actual (objectively measured) price and availability of foods. Prev Med 45, 41-48.

10. Allen F (2010) Health Psychology and Behaviour in Australia. Sydney: McGraw Hill Australia.

11. Swinburn B, Egger G \& Raza F (1999) Dissecting obesogenic environments: the development and application of a framework for identifying and prioritizing environmental interventions for obesity. Prev Med 29, 563-570.

12. Brug H (2008) Determinants of healthy eating: motivation, abilities and environmental opportunities. Fam Pract 25, 1-6.

13. Glanz K, Sallis JF, Saelens BE et al. (2005) Healthy nutrition environments: concepts and measures. Am J Health Promot 19, 330-333.

14. Burns CM \& Inglis AD (2007) Measuring food access in Melbourne: access to healthy and fast foods by car, bus and foot in an urban municipality in Melbourne. Health Place 13, 877-885.

15. Powell LM, Slater S, Mirtcheva D et al. (2007) Food store availability and neighborhood characteristics in the United States. Prev Med 44, 189-195.

16. Ball K, Timperio A \& Crawford D (2009) Neighbourhood socioeconomic inequalities in food access and affordability. Health Place 15, 578-585.

17. Morland K, Wing S, Diez Roux A et al. (2002) Neighborhood characteristics associated with the location of food stores and food service places. Am J Prev Med 22, 23-29.

18. Lovasi GS, Hutson MA, Guerra M et al. (2009) Built environments and obesity in disadvantaged populations. Epidemiol Rev 31, 7-20.

19. Winkler E, Turrell G \& Patterson C (2006) Does living in a disadvantaged area mean fewer opportunities to purchase fresh fruit and vegetables in the area? Findings from the Brisbane Food Study. Health Place 12, 306-319.

20. Winkler E, Turrell G \& Patterson C (2006) Does living in a disadvantaged area entail limited opportunities to purchase fresh fruit and vegetables in terms of price, availability, and variety? Findings from the Brisbane Food Study. Health Place 12, 741-748.

21. Inglis V, Ball K \& Crawford D (2005) Why do women of low socioeconomic status have poorer dietary behaviours than women of higher socioeconomic status? A qualitative exploration. Appetite 45, 334-343.

22. Pearce J, Witten K, Hiscock R et al. (2007) Are socially disadvantaged neighbourhoods deprived of health-related community resources? Int J Epidemiol 36, 348-355.

23. Thornton LE, Crawford DA \& Ball K (2010) Neighbourhood-socioeconomic variation in women's diet: the role of nutrition environments. Eur J Clin Nutr 64, 1423-1432.

24. Dillman D (1978) Mail and Telephone Surveys: The Total Design Method. New York: Wiley.

25. Pearce J, Day P \& Witten K (2008) Neighbourhood provision of food and alcohol retailing and social deprivation in urban New Zealand. Urban Policy Res 26, 213-227.

26. Australian Bureau of Statistics (1998) National Nutrition Survey User's Guide 1995. Canberra: ABS.

27. Peat J (2001) Health Science Research: A Handbook of Quantitative Methods. Sydney: Allen and Unwin.

28. Moore LV, Diez Roux AV \& Brines S (2008) Comparing perception-based and geographic information system (GIS)-based characterizations of the local food environment. J Urban Health 85, 206-216.

29. Drewnowski A (2009) Obesity, diets, and social inequalities. Nutr Rev 67, Suppl. 1, S36-S39.

30. Drewnowski A (2010) The cost of US foods as related to their nutritive value. Am J Clin Nutr 92, 1181-1188.

31. Cummins S \& Macintyre S (2009) Are secondary data sources on the neighbourhood food environment accurate? Case-study in Glasgow, UK. Prev Med 49, 527-528.

32. Lake AA, Burgoine T, Greenhalgh F et al. (2010) The foodscape: classification and field validation of secondary data sources. Health Place 16, 666-673.

33. Zenk SN, Schulz AJ, Lachance LL et al. (2009) Multilevel correlates of satisfaction with neighborhood availability of fresh fruits and vegetables. Ann Behav Med 38, 48-59. 